\title{
A neurosurgically reversible cause of respiratory dysfunction in rheumatoid arthritis
}

\author{
Keyoumars Ashkan, Adrian T H Casey, Nicholas P Hirsch, H Alan Crockard
}

A 69-year-old woman had been diagnosed with rheumatoid-factor-positive, antinuclearantibody-negative, erosive rheumatoid arthritis in 1971 at the age of 44 years. She was managed originally on a combination of adrenocorticotropin, given at intervals, and nonsteroidal anti-inflammatory drugs but, by 1973, also required prednisolone. In 1977 there was progression of erosive changes and betamethasone was substituted for prednisolone, with penicillamine being added in 1984. She also underwent ureteric transposition for chronic reflux in 1963 and had been on long-term nitrofurantoin.

The history of respiratory disease started in 1981 when she presented acutely with shortness of breath. She had experienced mild dyspnoea associated with productive cough in the preceding 6 weeks but had attributed it to an upper respiratory tract infection. She had smoked up to 20 cigarettes per day for 10 years but had stopped in 1960. Respiratory examination demonstrated reduced chest expansion and widespread late inspiratory crackles across both lung fields. Chest $\mathrm{X}$-ray showed bilateral reticulonodular shadows. Originally, these findings were thought to suggest rheumatoid-associated lung fibrosis. This diagnosis was, however, revised to a nitrofurantoinrelated fibrosis when grossly abnormal liver function tests and a positive antinuclear antibody results were obtained. She was managed by changing nitrofurantoin to co-trimoxazole, and a highdose course of prednisolone. On this combination her liver function tests returned to normal, but lung function assessments 6 weeks after the treatment indicated severe, predominantly restrictive, ventilatory defect (table). Her respiratory function continued to deteriorate and by 1984 she required oxygen at home. Further formal lung function testing became impossible because of poor respiratory reserve, and by 1985 , she needed oxygen tanks on each level of stairs.

In 1986 she complained of increasing neck pain associated with paraesthesia in all four limbs. Clinical examination confirmed pyramidal weakness and hyper-reflexia in the limbs. X-Rays of the cervical spine showed rheumatoid involvement of the atlantoaxial complex. A computed tomography (CT) myelogram was performed (figure).

Table Results of lung function tests performed before (1981) and after (1988) surgery for cervicomedullary decompression

The National Hospital

\begin{tabular}{|c|c|c|c|c|}
\hline & \multicolumn{2}{|c|}{ Before surgery } & \multicolumn{2}{|c|}{ After surgery } \\
\hline & Value & $\begin{array}{l}\% \\
\text { predicted }\end{array}$ & Value & $\begin{array}{l}\text { \% } \\
\text { predicted }\end{array}$ \\
\hline \multicolumn{5}{|l|}{ Forced expiratory volume in } \\
\hline $1 \mathrm{~s}\left(\mathrm{FEV}_{1}\right)$ & 0.32 & 13 & 1.35 & 61 \\
\hline Forced vital capacity (FVC) & 0.32 & 10 & 1.82 & 61 \\
\hline $\mathrm{FEV}_{1}:$ FVC & 100 & 128 & 74 & 99 \\
\hline \multicolumn{5}{|c|}{ Functional residual capacity } \\
\hline (1) & 1.28 & 39 & 3.15 & 98 \\
\hline Total lung capacity (1) & 1.88 & 35 & 4.42 & 82 \\
\hline Vital capacity (l) & 1.00 & 32 & 2.04 & 68 \\
\hline Residual volume (1) & 0.88 & 45 & 2.38 & 120 \\
\hline \multicolumn{5}{|l|}{ Diffusion capacity of lung } \\
\hline for CO (SI unit) & 3.3 & 41 & 5.34 & 72 \\
\hline \multicolumn{5}{|l|}{ Transfer coefficient (SI } \\
\hline
\end{tabular}



Figure CT myelogram through the upper cervical cord

Neurosurgery, Queen

Square, London

WC1N 3BG, UK

K Ashkan

A T H Casey

N P Hirsch

H A Crockard

Correspondence to $\mathrm{Mr}$ Alan Crockard

\section{Questions}

1 Name the structures labelled $a, b$, and $c$. What does the scan show?

2 How commonly does rheumatoid arthritis involve the cervical spine and what are the implications for respiratory function?

3 How would you treat this patient? 


\section{Answers}

QUESTION 1

The structures are: $a=$ anterior arch of Atlas; $\mathrm{b}=$ odontoid process; $\mathrm{c}=$ cervical cord. The scans shows compression at the upper cervical cord.

QUESTION 2

Involvement of the cervical spine in rheumatoid arthritis is only second in frequency to that of the metatarsophalangeal joints, occurring in up to $80 \%$ of patients. ${ }^{1}$ When it affects the atlantoaxial complex, compression of the cervical spinal cord and even cervicomedullary junction may occur. Apnoea and sudden death can arise as a consequence of the latter. ${ }^{2}$

\section{QUESTION 3}

The patient underwent transoral odontoidectomy and Ransford loop posterior cervical stabilisation in 1987. Postoperatively, her neurological symptoms, mobility and manual dexterity improved. The most striking effect, however, was upon her respiratory function. Within four weeks after the operation she noticed a reduction in her need to use supplementary home oxygen and by 6 months had totally abandoned it. This improvement was evident by the formal lung function test performed after the surgery (table). At 5-year follow-up her respiratory function has remained stable with no need for home oxygen, and the clinical and radiological examinations indicate satisfactory cervicomedullary decompression and stabilisation.

\section{Discussion}

Dyspnoea in the patient with rheumatoid disease is commonly the result of pulmonary, pleural or cardiac involvement by the disease or its treatment. ${ }^{3}$ However, respiratory dysfunction may also result from compression at the craniocervical junction due to atlantoaxial disease. This may result in an acute or progressive respiratory deterioration. ${ }^{4}$ Furthermore, lesions at this site have been shown to produce disordered breathing during sleep ${ }^{5}$ which may occur in the absence of daytime respiratory symptoms, focal brainstem signs or myelopathy. ${ }^{6}$ There are a number of mechanisms involved. These include direct compression of the medullary and pontine centres responsible for automatic respiratory drive and

1 Agarwal AK, Peppelman WC, Kraus DR, Eisenbeis CH. The cervical spine in rheumatoid arthritis. BMf 1993;306:79-80.

2 Davis FW, Markley HE. Rheumatoid arthritis with death from medullary compression. Ann Intern Med 1951;35:45161.

3 Jayson MIV, Grennan DM. Clinical features of rheumatoid arthritis. In: Weatherall DJ, Leadingham JGG, Warrell DA eds. Oxford Textbook of medicine, 2 nd edn. Oxford: Oxford University Press, 1987 ; 16.3-16.8.

\section{Learning point}

In patients with rheumatoid atlantoaxial disease, cervicomedullary compression may be the cause of respiratory dysfunction; surgical decompression of the craniocervical junction may lead to significant improvement in respiratory function

involvement of descending ventrolateral pathways resulting in depression of automatic control. In addition, angulation of the medulla and upper cervical cord may impair corticospinal tract and phrenic nerve nucleus function. Medullary compression may also contribute to a loss of chemoreceptor drive.

In this patient respiratory dysfunction may have resulted from a combination of pulmonary fibrosis and craniocervical compression. Indeed, her lung function tests predominantly indicated a restrictive pattern of respiratory dysfunction which may be due partly to interstitial pulmonary fibrosis, and partly to ventilatory failure secondary to cervicomedullary compression. The symptomatic improvement shortly after the craniocervical decompression, accompanied by a greater than two-fold increase in her vital capacity, however, highlight the important contribution of the latter.

The role of surgical intervention in patients with craniocervical compression and nocturnal respiratory dysfunction was illustrated by Howard et al who demonstrated resolution of pre-existing nocturnal desaturation following decompression. ${ }^{6}$ Here we present a patient with rheumatoid arthritis in whom surgical decompression of craniocervical junction led to significant improvement in the overall respiratory function. This case highlights the importance of identifying patients with rheumatoid atlantoaxial disease in whom it may represent an important but reversible cause of respiratory morbidity.

\section{Final diagnosis}

Rheumatoid compression of cervicomedullary junction.

Keywords: rheumatoid arthritis; respiratory dysfunction; cervicomedullary compression

4 Fish DR, Howard RS, Wiles CM, Symon L. Respiratory arrest: a complication of cerebellar ectopia in adults. $\mathcal{F} \mathrm{Neu}$ rol Neurosurg Psychiatry 1988;51:714-5.

5 Fisher MA, Casey LC, Ellman MH, Perlick SJ. Sleep apnea due to odontoid brainstem compression in a patient with rheumatoid arthritis. Neurology 1986;36:163.

6 Howard RS, Henderson F, Hirsch NP, Stevens JM, Kendall BE, Crockard HA. Respiratory abnormalities due to craniovertebral junction compression in rheumatoid disease. Rheum Dis 1994:53:134-6. 